\title{
Asymptomatic Onset of Autoimmune Hepatitis does not Mean Better Prognosis
}

\section{Claudine Lalanne*, Paolo Muratori, Marco Lenzi and Luigi Muratori}

Department of Medical and Surgical Sciences, Centre for the Study and Treatment of Autoimmune Diseases of the Liver and Biliary System, Policlinico di S.Orsola, Bologna, Italy

${ }^{*}$ Corresponding author: Claudine Lalanne, Department of Medical and Surgical Sciences, Centre for the Study and Treatment of Autoimmune Diseases of the Liver and Biliary System, Policlinico di S.Orsola, Bologna, Italy, Tel: +39-051-2143748; Fax:+39-051-2144886; E-mail: claudine.lalanne@studio.unibo.it

Received date: March 21, 2016; Accepted date: June 09, 2016; Published date: June 11, 2016

Citation: Lalanne C, Muratori P, Lenzi M, Muratori L (2016) Asymptomatic Onset of Autoimmune Hepatitis Does Not Mean Better Prognosis. J Autoimmune Disord 2: 4. doi: 10.4172/2471-8153.100020

Copyright: (C) 2016 Lalanne C, et al. This is an open-access article distributed under the terms of the Creative Commons Attribution License, which permits unrestricted use, distribution, and reproduction in any medium, provided the original author and source are credited.

\section{Abstract}

Clinical presentation of autoimmune hepatitis can be either acute or chronic or totally asymptomatic. The aim of this study was to assess the impact of asymptomatic onset on AlH natural history. Ninety asymptomatic patients were retrospectively compared to 215 with symptoms. Asymptomatic presentation was characterized by lower histological grading and lower mean levels of aminotransferases, bilirubin, gamma-globulins, IgG and anti-dsDNA. On the other hand, asymptomatic patients were more often positive for anti-LKM1 autoantibodies and were more often affected by extrahepatic autoimmune conditions, particularly thyroid and skin disorders.

Despite these differences, asymptomatic and symptomatic subjects had similar response to immunosuppressive treatment and comparable prognosis, with a low but considerable rate of progression (23\%) towards cirrhosis or its complications.

\section{Keywords:}

Autoimmune;

Liver;

Asymptomatic;

Autoantibodies; Steroid; Cirrhosis

\section{Abbreviations}

AlH: Autoimmune Hepatitis; PBC: Primary Biliary Cholangitis; PSC: Primary Sclerosing Cholangitis; EASL: European Association for the Study of the Liver; IIF: Indirect Immunofluorescence (IIF); ANA: Antinuclear Antibodies (ANA); SMA: Anti-smooth Muscle Antibodies (SMA); anti-LKM: AntiLiver/Kidney Microsomal Antibodies; anti-dsDNA: Anti-Double Strand DNA antibodies; ALT: Alanine Aminotransferase; AST: Aspartate Aminotransferase; ALP: Alkaline Phosphatase; GGT: Gamma-Glutamyl Transpeptidase; IgG: Immunoglobulin G; INR: International Normalized Ratio; HLA: Human Leukocyte Antigen; ULN: Upper Limit of Normal.

\section{Introduction}

Autoimmune hepatitis is a rare form of chronic liver inflammation due to an unexplained loss of immune tolerance towards self-hepatocytes [1]. If not treated with immunosuppressive drugs, such as corticosteroids and azathioprine, AlH may lead to cirrhosis and eventually to endstage liver disease [2]. It is known as a disorder of very young or middle-aged women, but people of any age, gender and ethnic group can be affected [3].

Typical features of $\mathrm{AlH}$ are hypergammaglobulinemia (irrespective of the presence of cirrhosis), circulating autoantibodies and interface hepatitis, but none of them is pathognomonic.

Not even clinical presentation is disease specific and that is another reason why the diagnosis is particularly challenging. About one third of patients present with an acute - rarely fulminant - course akin to viral hepatitis with jaundice and highly elevated liver enzymes. Another third of subjects have an insidious or chronic onset with a long history of unspecific symptoms like fatigue, malaise, anorexia, and weight loss, mild abdominal pain in the right upper quadrant, arthralgia (preferentially small joints), amenorrhea, maculopapular skin rash and unexplained low-grade fever. Last third of patients is completely asymptomatic. In this case $\mathrm{AlH}$ is diagnosed accidentally, due to routine laboratory tests revealing unexpectedly elevated liver enzymes [3-5].

According to previous studies by Kogan et al. and Feld et al. $[6,7]$ asymptomatic patients are thought to have a milder disease and a better prognosis, whether or not they receive immunosuppressive therapy. On account of this, last EASL guidelines on the management of AlH recommend treating only patients with symptoms and/or advanced fibrosis; as for asymptomatic subjects with low-grade disease activity and mild fibrosis, therapy can be deferred until the development of symptoms or signs of disease progression [3].

This issue is still up for debate. In our experience (in Bologna, Italy) it appears that clinical outcomes of asymptomatic and symptomatic patients are almost identical. 
These data have been recently reported in a full paper [8] that is here summarized as a short communication.

\section{Methods}

We retrospectively evaluated 305 subjects followed up at our Centre between 1994 and 2013 with a diagnosis of $\mathrm{AlH}$ according to the Simplified Scoring System of the International Autoimmune Hepatitis Group $[9,10]$. Two hundred and twentynine patients $(75 \%)$ had a score of definite $\mathrm{AlH}$, in the remainder $76(25 \%)$ the diagnosis was probable.

Patients with features of overlapping primary biliary cholangitis or primary sclerosing cholangitis were excluded, as well as those affected by viral, toxic, metabolic or genetic liver diseases. The study was approved by our Institutional Review Board.

Enrolled patients had undergone routine biochemistry tests, autoantibodies screening (ANA, SMA and anti-dsDNA by IIF. Anti-LKM1 by IIF and then by Western immunoblot) and abdominal ultrasound scan at diagnosis and repeatedly during follow-up. One hundred and eighty patients (59\%) had been tested also for class I and class II HLA susceptibility alleles (DR3 and DR4). A liver biopsy obtained prior to treatment initiation was available in 248 out of 305 subjects (81.3\%). Every patient had been given standard therapy (corticosteroids with or without azathioprine), which had been slowly tapered to the minimal maintenance dose and withdrawn, whenever possible.

Statistical analysis was performed through Fisher's exact test (categorical variables), unpaired $t$ test or KolmogorovSmirnov test (continuous data), Kaplan-Meier and log-rank Mantel-Cox test (cumulative rate of clinical outcomes) [Wizard 1.6.5 and Graph Pad Prism 3.0 for Mac OS X, La Jolla, California]; $p$ values $<0.05$ were considered significant.

\section{Results}

Two hundred and fifteen (70.5\%) out of 305 patients had a symptomatic onset, either acute $(66.5 \%$ of 215$)$ or insidious $(33.5 \%)$, the remainder 90 (29.5\%) were asymptomatic. They shared the same age and sex distribution (clear female predominance in both groups).

At baseline asymptomatic patients had significantly lower levels of aminotransferases (AST and ALT), bilirubin, ALP, GGT, gamma-globulins $\mathrm{G}$ and IgG, but similar levels of hepatocellular synthetic indices such as INR, albumin and cholesterol (Table 1).

Asymptomatic subjects showed lower histological grading and comparable staging (248/305 pre-treatment biopsies), as well as the same prevalence of cirrhosis (Table 1 ).

ANA, SMA and class I and II HLA alleles were equally distributed in the cohort; on the contrary, anti-LKM1 and antidsDNA were respectively more and less frequent in the asymptomatic compared to the symptomatic group (Table 1).
Extrahepatic autoimmune diseases, especially autoimmune thyroid and skin disorders (Hashimoto's thyroiditis or Grave's disease, vitiligo, psoriasis), were associated significantly more often with the asymptomatic phenotype (Table 1).

Table 1: Comparison of demographic, clinical, biochemical, serological and histological features of asymptomatic and symptomatic AlH patients at disease onset (continuous data expressed as means \pm standard deviation).

\begin{tabular}{|c|c|c|c|}
\hline & $\begin{array}{c}\text { Asymptoma } \\
\text { tic onset } \\
\text { (90 pts) }\end{array}$ & $\begin{array}{l}\text { Symptom } \\
\text { atic onset } \\
(215 \mathrm{pts})\end{array}$ & $p(<0.05)$ \\
\hline Age $(y)$ & $38.6 \pm 19.8$ & $\begin{array}{c}37.9 \pm \\
19.4\end{array}$ & ns \\
\hline Female sex (\%) & 82.2 & 76.7 & ns \\
\hline Follow up (mo) & $79 \pm 64$ & $73 \pm 66$ & ns \\
\hline AST (xULN) & $6.52 \pm 7.39$ & $\begin{array}{c}19.71 \pm \\
16.65\end{array}$ & $<0.001$ \\
\hline ALT (xULN) & $7.90 \pm 8.07$ & $\begin{array}{c}23.87 \pm \\
18.77\end{array}$ & $<0.001$ \\
\hline GGT (xULN) & $2.51 \pm 3.38$ & $\begin{array}{c}3.81 \pm \\
3.46\end{array}$ & 0.01 \\
\hline ALP $(x \cup L N)$ & $1.1 \pm 0.7$ & $1.7 \pm 1.5$ & 0.003 \\
\hline Bilirubin (mg/dL) & $1.4 \pm 1.4$ & $8.6 \pm 10.4$ & $<0.001$ \\
\hline Gamma-globulins (g/L) & $22.1 \pm 8.6$ & $26 \pm 10.7$ & 0.01 \\
\hline Albumin $(\mathrm{g} / \mathrm{L})$ & $41.2 \pm 6.9$ & $39.2 \pm 9.1$ & ns \\
\hline INR & $1.1 \pm 0.1$ & $1.2 \pm 0.3$ & ns \\
\hline Cholesterol (mg/dL) & $171 \pm 47$ & $159 \pm 51$ & ns \\
\hline $\lg G(x \cup L N)$ & $1.33 \pm 0.57$ & $\begin{array}{c}1.60 \pm \\
0.70\end{array}$ & 0.011 \\
\hline $\operatorname{IgA}(x \cup L N)$ & $0.73 \pm 0.37$ & $\begin{array}{c}0.83 \pm \\
0.51\end{array}$ & ns \\
\hline $\operatorname{lgM}(x \cup L N)$ & $0.83 \pm 0.42$ & $\begin{array}{c}0.85 \pm \\
0.62\end{array}$ & ns \\
\hline Histological grading & $7 \pm 2.5$ & $9 \pm 2.9$ & $<0.001$ \\
\hline Histological staging & $2.3 \pm 1.1$ & $2.2 \pm 1.2$ & ns \\
\hline Cirrhosis (\%) & 10 & 15.5 & ns \\
\hline ANA (\%) & 50.7 & 61.4 & ns \\
\hline SMA (\%) & 46.5 & 57.5 & ns \\
\hline Anti-LKM1 (\%) & 26.8 & 13.1 & 0.006 \\
\hline Anti-LC1 (\%) & 15.9 & 12.1 & ns \\
\hline Anti-dsDNA (\%) & 9.8 & 31.7 & 0.001 \\
\hline HLA-DRB1*03 (\%) & 29 & 41.9 & ns \\
\hline HLA-DRB1*04 (\%) & 27.4 & 24.1 & ns \\
\hline HLA-DRB1*07 (\%) & 16.7 & 25.9 & Ns \\
\hline $\begin{array}{c}\text { Extrahepatic Autoimmune } \\
\text { Diseases (\%) }\end{array}$ & 43.3 & 27.9 & 0.009 \\
\hline $\begin{array}{l}\text { Autoimmune Thyroid Diseases } \\
(\%)\end{array}$ & 26.7 & 12.6 & 0.003 \\
\hline
\end{tabular}


Autoimmune Skin Disorders (\%)

\section{9}

2.3

0.01

Asymptomatic and symptomatic patients had analogous rates of treatment response as well as relapse after immunosuppression withdrawal ( $80 \%$ vs $81 \%$ ) (Table 2 ). About $60 \%$ of patients achieved a complete response $160.1 \%$ asymptomatic vs $56.5 \%$ symptomatic), regarded as absence of symptoms and normalization of both aminotransferases and IgG on low dose maintenance therapy. Despite higher immunosuppression, a quarter of subjects ( $25.8 \%$ vs $25.5 \%$ ) reached only a partial response (transaminases levels $<2$ fold the ULN), whereas the others failed to respond $(14.1 \%$ vs $18 \%)$.

Table 2: Treatment response and clinical outcomes according to asymptomatic and symptomatic onset.

\begin{tabular}{|c|c|c|c|}
\hline & $\begin{array}{r}\text { Asymptomatic } \\
\text { onset (90 pts) }\end{array}$ & $\begin{array}{c}\text { Symptomatic } \\
\text { onset (215 pts) }\end{array}$ & $\mathrm{p}<0.05$ \\
\hline Complete response & $60.10 \%$ & $56.50 \%$ & $\mathrm{~ns}$ \\
\hline Partial response & $25.80 \%$ & $25.50 \%$ & $\mathrm{~ns}$ \\
\hline Treatment failure & $14.10 \%$ & $18 \%$ & $\mathrm{~ns}$ \\
\hline Relapse & $80 \%$ & $81 \%$ & $\mathrm{~ns}$ \\
\hline Remission & $76.4 \%(55 / 72)$ & $\begin{array}{c}77.8 \% \\
(151 / 194)\end{array}$ & $\mathrm{ns}$ \\
\hline Progression: & $23.6 \%(17 / 72)$ & $\begin{array}{c}22.2 \% \\
(43 / 194)\end{array}$ & $\mathrm{ns}$ \\
\hline to cirrhosis & $18.5 \%(12 / 65)$ & $\begin{array}{c}12.2 \% \\
(20 / 164)\end{array}$ & $\mathrm{ns}$ \\
\hline $\begin{array}{c}\text { to cirrhosis' } \\
\text { complications }\end{array}$ & $71.4 \%(5 / 7)$ & \begin{tabular}{c}
$76.7 \%(23 / 30)$ \\
\hline
\end{tabular}
\end{tabular}

Two hundred and sixty six patients (87\%), 72 asymptomatic and 194 symptomatic, were followed up for at least 6 months; 12 subjects had a shorter follow up time and the remainder 27 dropped out. Two hundred and six patients $(77.4 \%)$ achieved remission, while 60 (22.6\%) developed either cirrhosis (14\%: 32 out of 229 non-cirrhotic subjects) or its complications, such as ascites, variceal bleeding, hepatic encephalopathy, hepatocellular carcinoma, death from liver failure or orthotopic liver transplant (75.6\%: 28 out of 37 cirrhotic patients). Disease progression occurred as frequently in asymptomatic $(23.6 \%)$ as in symptomatic patients $(22.2 \%)$. In the asymptomatic group, 12 of 65 non-cirrhotic patients developed cirrhosis (18.5\%) and 5 out of 7 cirrhotic subjects experienced at least one complication (71.4\%). In the symptomatic group progression to cirrhosis or its complications occurred respectively in 20/164 (12.2\%) and $23 / 30(76.7 \%)$ patients.

According to the initial stage, liver disease progressed earlier and more frequently in cirrhotic than in non-cirrhotic subjects, either symptomatic or not.

\section{Discussion}

The aim of this study was to assess the impact of asymptomatic presentation on the natural history of autoimmune hepatitis. Indeed, our cohort included the greatest number of asymptomatic patients (90) analysed so far.

No differences were detected in regard to female preponderance and age distribution, with a peak in the second and another one between the fourth and the fifth decade.

In line with other reports [6,7], we found asymptomatic presentation to be characterized by milder biochemical and histological activity, with lower levels of transaminases, bilirubin, gamma-globulins and IgG, as well as lower histological grading. Nonetheless, prevalence of cirrhosis at the time of diagnosis was similar between asymptomatic $(10 \%)$ and symptomatic (15.5\%) patients. Furthermore, we observed disease progression in about $23 \%$ of the study population, whatever the presentation $(23.6 \%$ asymptomatic and $22.2 \%$ symptomatic) and despite the fact that asymptomatic patients had been treated equally to the others. These data conflict with the common opinion of asymptomatic $\mathrm{AlH}$ as an indolent disease, which is based mainly on two previous studies. One, by Kogan et al. [6], compared 23 asymptomatic to 45 symptomatic subjects and detected lower frequency of cirrhosis at baseline, higher rate of response achieved earlier and with lower doses of steroids -, lower rate of therapy-related adverse events and lower frequency of disease progression. In the second study Feld et al. [7] analysed a cohort of 124 AlH patients, 93 with symptoms at diagnosis and 31 without. Asymptomatic patients appeared to have the same excellent 10 -year survival as the symptomatic subjects $(80 \%$ vs $83.8 \%$ ), despite not being treated in most cases $(51.6 \%)$.

In our asymptomatic patients disease progression occurred even if they received immunosuppressive treatment. It happened earlier and more frequently to cirrhotic patients, since cirrhosis is a known predictor of poor prognosis $[1,7]$, but even non-cirrhotic patients were at risk, as much as the symptomatic ones.

In our opinion, this is a strong indication to treat every patient with autoimmune hepatitis, unless there are severe contraindications due to active infectious disease, malignancy, poorly controlled diabetes or hypertension.

It must be also an indication to actively seek AlH in patients with extrahepatic autoimmune disorders (EADs). In keeping with previous findings [2,11], this study confirms a considerable association between $\mathrm{AlH}$ and other autoimmune diseases, especially those involving thyroid and skin. Asymptomatic patients seem to be more likely affected by EADs (43.3\% vs $27.9 \%$, p 0.009). It might be a selection bias, but it reminds us to extend to the liver the panel of laboratory tests performed in patients with various autoimmune conditions. 


\section{Conclusions}

Asymptomatic and symptomatic AlH patients differ in terms of laboratory findings, such as lower mean levels of alanine aminotransferase and bilirubin, but eventually they seem to share the same clinical outcomes and equally benefit from steroid therapy. Hence, it is recommended to screen for autoimmune hepatitis any patient with extrahepatic autoimmune conditions, particularly thyroid and skin disorders, which are significantly associated with asymptomatic $\mathrm{AlH}$.

\section{References}

1. Kirstein MM, Metzler F, Geiger E, Heinrich E, Hallensleben $M$, et al. (2015) Prediction of short- and long-term outcome in patients with autoimmune hepatitis. Hepatology 62: 1524-1535.

2. Wong GW, Heneghan MA (2015) Association of Extrahepatic Manifestations with Autoimmune Hepatitis. Dig Dis 33: 25-35.

3. European Association for the Study of the Liver (2015) EASL Clinical Practice Guidelines: Autoimmune Hepatitis. J Hepatol 63: 971-1004.

4. Gatselis NK, Zachou K, Koukoulis GK, Dalekos GN (2015) Autoimmune hepatitis, one disease with many faces: Etiopathogenetic, clinico-laboratory and histological characteristics. World J Gastroenterol 21: 60-83.
5. Ferrari R, Pappas G, Agostinelli D, Muratori P, Muratori L, et al. (2004) Type 1 autoimmune hepatitis: patterns of clinical presentation and differential diagnosis of the "acute" type. Q J Med 97: 407-412.

6. Kogan J, Safadi R, Ashur Y, Shouval D, Ilan Y (2002) Prognosis of symptomatic versus asymptomatic autoimmune hepatitis: a study of 68 patients. J Clin Gastroenterol 35: 75-81.

7. Feld JJ, Dinh H, Arenovich T, Marcus VA, Wanless IR, et al. (2005) Autoimmune hepatitis: Effect of symptoms and cirrhosis on natural history and outcome. Hepatology 42: 53-62.

8. Muratori P, Lalanne C, Barbato E, Fabbri A, Cassani F, et al. (2016) Features and Progression of Asymptomatic Autoimmune Hepatitis in Italy. Clin Gastroenterol Hepatol 14: 139-146.

9. Hennes EM, Zeniya M, Czaja AJ, Parés A, Dalekos GN, et al. (2008) Simplified criteria for the diagnosis of autoimmune hepatitis. Hepatology 48: 169-176.

10. Muratori P, Granito A, Pappas G, Muratori L (2009) Validation of simplified diagnostic criteria for autoimmune hepatitis in Italian patients. Hepatology 49: 1782-1783.

11. Muratori P, Fabbri A, Lalanne C, Lenzi M, Muratori L (2015) Autoimmune liver disease and concomitant extrahepatic autoimmune disease. Eur J Gastroenterol Hepatol 27: 1175-1179. 